\title{
Preserve the Uterus For Placenta Accreta Spectrum Overlying Cesarean Scar: a Step-by-step Surgical Technique
}

\author{
Tao $\mathrm{Li}^{1}$, Yilei $\mathrm{Li}^{1}$, Wen Peng ${ }^{1}$, and Hongmei Wang ${ }^{1}$ \\ ${ }^{1}$ Shandong Provincial Hospital
}

August 17, 2020

\begin{abstract}
Surgery for placenta accreta spectrum overlying the cesarean scar is challenging for excessive blood loss. Elective cesarean hysterectomy without disrupting the placenta was the generally accepted approach, however, with high maternal morbidity, loss of fertility and psychological trauma. Innovative approaches are being investigated for uterine preservation. A step-bystep surgical technique was developed in our group and has been used to preserve the uterus in all severe cases with placenta increta/percreta overlying the cesarean scar. The procedure details and the surgical outcomes are provided.
\end{abstract}

\section{Introduction:}

Placenta accreta spectrum (PAS) covers a spectrum of abnormally invasive placentation including placenta accreta, increta and percreta. The incidence of PAS with placenta previa has been increasing as the consequence of the world-wide rising cesarean section (CS) rate, from 1 in 4000 deliveries in the 1970s to 1 in 500 recently. ${ }^{1}$ The major problem of PAS is severe and sometimes life-threatening hemorrhage, which results in massive blood transfusion, coagulopathy, hysterectomy, need for re-operation, even maternal death. The optimal surgical management for PAS remains uncertain. Elective cesarean hysterectomy without disrupting the placenta, was the most generally accepted approach. ${ }^{2-4}$ Delayed hysterectomy after cesarean delivery with the placenta left in situ, was also reported to decrease blood loss. ${ }^{5-6}$ However, this approach need two major surgeries, and the delay poses a risk of bleeding or infection that may require emergency surgery.

Due to the high maternal morbidity and surgical complications, in addition to loss of fertility and its accompanying psychological trauma caused by cesarean-hysterectomy, innovative approaches are being investigated for uterine preservation. These methods include preoperative arterial balloon ${ }^{7,8}$ pelvic arterial ligation, ${ }^{3}$ compression sutures,${ }^{8-10}$ local resection and uterine reconstruction. ${ }^{11,12}$ However, the successful rate of uterine preservation in literatures varied greatly, and the sample size of studies with total uterine preservation are not large enough for an reassuring consensus.

Under this circumstances, a step-by step protocol was developed in our group and has been used for uterine preservation in PAS patients. The procedure details and the surgical outcomes are provided.

\section{Methods:}

\section{Diagnose of placenta accreta}

Patients diagnosed as PAS overlying the cesarean scar were included. A thorough perioperative sonography was performed to determine the invasion of the placenta, the length and shape of cervix. Cervical involvement was determined when the placenta covered the internal os and blood flow could be detected inside the shortened cervix canal (length $<2 \mathrm{~cm}$ ). Those with two or more signs of the following were considered to be severe and preoperative aortic balloon was suggested: two or more times of previous CS; cervical 
involvement; complete placenta previa; placental lacunae and turbulence, and loss of myometrial interface with width $>3 \mathrm{~cm}$; bladder wall interruption and uterovescical hypervascularity.

The severity of placenta invasion was evaluated intraoperatively. Placenta accreta: manual removal of placenta required; Placenta increta: part of the placenta need cut with sissors, the lefted uterine wall was rather thinner than those around; Placenta percreta: placenta tissue seen to have invaded through the serosa of the uterus with hypervascularity, or a clear surgical plane cannot be identified between the bladder and uterus. Placenta increta/percreta with extension over $1 / 3$ of the placenta, or with cervical involvement was determined to be severe.

\section{Description of Intervention}

Before surgery, the $\mathrm{Hb}$ level was brought up to $>100 \mathrm{~g} / \mathrm{L}$ by blood transfusion. The possibility of intractable blood loss and hysterectomy has been discussed with the family and written consent was assigned. Combined lumbar anesthesia was applied in those without any risking factors according to preoperative sonography, and general anesthesia was used for the others.

We choose to open on the previous scar to avoid a cruciate incision except when the original transverse incision is very low. A incision sleeve is helpful for a fairly good exposure of the surgical field in both transverse and longitudinal incision. General anesthesia was initiated after all the elements were in position.

1: Open the utero-vesical serosa and try to separate the bladder from the lower uterine segment (video S1). There's always engorged aberrant vessels under the utero-vesical fold, which should be opened elaborately. Pushing the bladder down enough to below the level of the uterine artery will help in later compression sutures. Sometimes it's difficult for tense adhesion and bleeding, the bladder might be broken. Therefore this procedure had to be performed after delivery with sharp dissection.

2: Block the bleeding rightly after the baby is delivered and remove the placenta. Huge blood loss usually begins with the delivery, especially when the placenta was mainly located at the anterior wall. Try to avoid the placenta when cutting the uterus. If it's not possible, cut the placenta with hand decisively to deliver the baby as soon as possible and leave it to the second assistant. Exteriorize the uterus from the pelvic cavity and grasp the lower uterine segment tightly to block bleeding with the operator's left hand (The operator stands at the right side of the patient). Bleeding usually attenuate dramatically. Detach the placenta in bulk with right hand, and then clamp the lower uterine segment with two ovum forceps from each side (Figure 1). Bleeding will attenuate remarkably, clean the remaining placenta. (video S2)

3: Ligate the bilateral uterine artery and the lateral part of the lower uterine segment (video S2). With the uterus being distracted upward by the assistant, use the left hand to replace one of the ovum forceps, push the bladder down enough with the right hand, and do suture A (Figure 1, Figure 2) to ligate the uterine artery and compress the lateral lower uterine segment simultaneously. Do it for three times at $2-3 \mathrm{~cm}$ interval upward on each side.

4: Do sutures on the anterior lower uterine segment (suture B) (Figure 2) (video S2). With the index finger or the middle finger of the left hand as indicator, do sutures on the anterior lower uterine segment. By pushing the bladder down and outward enough, perform the suture on very low level without damaging the bladder or the ureter.

5: Do horizontal sutures on the posterior lower uterine segment (suture C) (Figure 3) (video S2). If bleeding continues from the lower part, do several horizontal sutures with large steps at $2-3 \mathrm{~cm}$ interval upward with the index finger of the left hand as indicator.

6: Cut around the uterine incision if the uterine wall has been penetrated or very thin (Figure 2) (video S2). This will also decrease the volume of the uterus and therefore help in hemostasis. Furthermore, the healing of the incision would be better with thicker sides.

7: Usually another long step suture is needed on each end of the uterine incision (suture D) (Figure 2) (video S2). Additional local haemostatic sutures on the uterine wall may be needed. In very difficult cases, a long 
verticle suture running through the anterior and posterior lower segment on each side is necessary.

Complete hemostasis can be achieved in most situations with step 1-7.

8: Do suspending sutures on the uterine body (Figure 1, Figure 2). Bleeding from the upper part of the uterus may occur for uterine inertia and coagulopathy after huge blood loss. Suture E will work definitely. Compression on the uterus body by the assistant to tighten the suture is indispensable. Usually five stiches for term pregnancy uterus and three stiches for smaller uterus are needed. The longitudinal sutures will perfectly compress the upper part of the uterus and allow the drainage of occult intrauterine hemorrhage. The tiny space on the top of the uterus is used to prevent the incarceration of the bowl when the uterus shrinks later.

Then compression sutures has been performed in the entire uterus, and complete hemostasis can be achieved. Close and embed the uterine incision carefully. Do not stretch strongly if large area around the incision has been cut. Finally close the utero-vesical serosa after comprehensive hemostasis on the bladder.

\section{Results:}

A consecutive cohort of 116 patients were treated with this technique (Table 1) from 2016.1 - 2019.12. The overall estimated blood loss (EBL) was $1656.9 \pm 1376.6 \mathrm{ml}$ with an average surgical time of $2.2 \pm 0.8 \mathrm{~h}$. The uterus was preserved successfully in all patients. Suspending suture has been performed in 16/116 (13.8\%) patients. Damage to the bladder occurred in 13/116 (11.2\%) patients and were repaired instantly during the surgery, followed with good recovery after bladder irrigation and drainage. All patients recovered well without severe complications such as damage to the ureter, serious infection, or ischemic uterine necrosis.

In the 44 severe cases determined during surgery, only 21 patients received perioperative aortic balloon. The EBL in the balloon group $(\mathrm{n}=21)$ was slightly lower than those without balloon $(\mathrm{n}=23)(2913.0 \pm 1773.6$ $\mathrm{ml}$ versus $2809.5 \pm 1212.8 \mathrm{ml})$, however, the difference was not significant $(\mathrm{P}=0.824)$.

\section{Discussion:}

We present a step-by-step surgical protocol to preserve the uterus for patients with PAS overlying the cesarean scar. Any possible bleeding area including the placenta bed can be perfectly compressed with ideal efflux canal. Additionaly, with immediate blockage of hemorrhage rightly after delivery, and dissection of very thin part of the involved area, this protocol allowed for thorough clean of the placenta tissue. Finally, most surgeries was accomplished on the previous cesarean scar, a long attitude incision which was needed to deliver the baby without touching the placenta, can be avoided.

With this technique, make sure to do sutures without damaging the ureter by pushing the bladder downward and outward from the anterior lower uterine segment and cervix. To achieve hemostasis in the uterus, large step sutures are suggested. With large step sutures, hemostasis can be achieved within a shorter time by covering larger area and decreasing the uterine volume, the possiblility of local ischemic necrosis is also minimized. Enough blood preparation before surgery, timely infusion of blood products according to EBL is also essential to fulfill the process. Without timely supply, instant blood loss will lead to uterine inertia and intractable bleeding, and suspending sutures will be the only effective procedure to cut this vicious cycle.

Preoperative aortic balloon has been proved to be effective to reduce bleeding and shorter surgical time. ${ }^{7}$ In this cohort, in the 44 patients determined to be severe, the EBL in the balloon group was not significantly lower than those without balloon. The reason maybe that following this protocol, the immediate blockage of hemorrhage with hand and oval clamps resembled the effect of blockage of the aortic balloon. Considering the complications of the balloon technique, such as aortic rupture, ischemia and thrombosis in the lower extremities, reperfusion injury, the choose of balloon should be more conservative. ${ }^{13}$

The limitations of this research lies in its retrospective nature, and the shortage of standard diagnostic criteria on the severity of placenta invasion.

\section{Conclusion:}


Uterine preservation with one surgery is possible in PAS patients with placenta increta/percreta overlying the cesarean scar.

\section{Acknowledgement:}

We would like to thank Zeqi Wang for the artwork, we than Dr Jing Chai for the video.

\section{Disclosure of Interests:}

The authors have no conflicts to disclose.

\section{Contribution to authorship:}

HW conceived and performed the protocol. TL and WP drafted the manuscript, HW and YL contributed to editing and reviewing the manuscript.

\section{Ethical Approval:}

None required.

\section{Funding:}

There was no funding for this publication.

\section{References:}

1. Jauniaux E, Bhide A, Kennedy A, Woodward P, Hubinont C, Collins S, et al. FIGO consensus guidelines on placenta accreta spectrum disorders: Prenatal diagnosis and screening. Int J Gynaecol Obstet. 2018 Mar;140(3):274-80.

2. Silver RM, Branch DW. Placenta Accreta Spectrum. N Engl J Med. 2018 Apr 19;378(16):1529-36.

3. Sentilhes L. Maternal outcome after conservative treatment of placenta accreta. Obstet Gynecol. 2010.

4. Turan OM, Shannon A, Asoglu MR, Goetzinger KR. A novel approach to reduce blood loss in patients with placenta accreta spectrum disorder. J Matern Fetal Neonatal Med. 2019 Aug 27:1-10

5. Zuckerwise LC, Craig AM, Newton JM, Zhao S, Bennett KA, Crispens MA. Outcomes following a clinical algorithm allowing for delayed hysterectomy in the management of severe placenta accreta spectrum. Am J Obstet Gynecol. 2020 Feb;222(2):179 e1-79 e9.

6. Chevalier G, Devisme L, Coulon C. [Placenta accreta spectrum disorder: Management and morbidity in a French type-3 maternity]. Gynecol Obstet Fertil Senol. 2020 Mar 12.

7. Wei Y, Luo J, Luo D. Comparison of Efficacy between Internal Iliac Artery and Abdominal Aorta Balloon Occlusions in Pernicious Placenta Previa Patients with Placenta Accrete. Gynecol Obstet Invest. 2019;84(4):343-49.

8. Zhao B, Lv M, Dong T, Chen Y, Xi F, Lv W, et al. Transverse parallel compression suture: a new suturing method for successful treating pernicious placenta previa during cesarean section. Arch Gynecol Obstet. 2020 Feb;301(2):465-72

9. Mohamed MA, Mohammed AH. Parallel vertical compression sutures to control bleeding in cases of placenta previa and accreta. J Matern Fetal Neonatal Med. 2019 Feb;32(4):641-45.

10. Li GT, Li GR, Li XF, Wu BP. Funnel compression suture: a conservative procedure to control postpartum bleeding from the lower uterine segment. BJOG. 2016 Jul;123(8):1380-5

11. Palacios-Jaraquemada JM, Fiorillo A, Hamer J, Martinez M, Bruno C. Placenta accreta spectrum: a hysterectomy can be prevented in almost $80 \%$ of cases using a resective-reconstructive technique. J Matern Fetal Neonatal Med. 2020 Jan 26:1-8. 
12. Zhao X, Tao Y, Du Y, Zhao L, Liu C, Zhou Y, et al. The application of uterine wall local resection and reconstruction to preserve the uterus for the management of morbidly adherent placenta: Case series. Taiwanese Journal of Obstetrics and Gynecology. 2018;57(2):276-82

13. Sentilhes L, Kayem G, Chandraharan E, Palacios-Jaraquemada J, Jauniaux E, Diagnosis FPA, et al. FIGO consensus guidelines on placenta accreta spectrum disorders: Conservative management. Int J Gynaecol Obstet. 2018 Mar;140(3):291-98.

Figure 1. Clamp the lower uterine segment with two ovum forceps from each side to block hemorrhage.

Figure 2. Anterior view of compression sutures on the whole uterus.

Figure 3. Posterior view of compression sutures on the whole uterus.

Table 1. Characteristics, surgical details and outcome of 116 PAS patients with placenta overlying cesarean scar undergoing the protocol.

Table 1 Characteristics, surgical details and outcome of 116 PAS patients with placenta overlying cesarean scar undergoing the protocol.

\begin{tabular}{ll}
\hline Variables & Statistics \\
Mean maternal age in years (SD) & $33.3 \pm 5.1$ \\
Mean gestational age at delivery (SD) & $35.9 \pm 2.2$ \\
Number of previous cesarean sections $(1 / 2 / 3)$ & $95 / 19 / 2(81.9 / 16.4 / 1.7 \%)$ \\
Neonatal Birth Weight (g) & $2774.1 \pm 522.7$ \\
Hospital stay after surgery (d) & $4.4 \pm 2.1$ \\
Aortic balloon occlusion & $23 / 116(19.8 \%)$ \\
Estimated blood loss (ml) & $1656.9 \pm 1376.6$ \\
Surgical time (h) & $2.2 \pm 0.8$ \\
Suspending suture(Y/N) & $16 / 116(13.8 \%)$ \\
Additional operations* & 23 \\
Cervical involvement & $22 / 116(19.0 \%)$ \\
Placenta accreta/increta/deep inceta/percreta & $16 / 73 / 27(13.8 / 63.0 / 23.3 \%)$ \\
Extension of placenta increta (None/0-1/3/>1/3) & $16 / 51 / 49(13.8 / 44.0 / 42.2 \%)$ \\
Hysterectomy & 0 \\
Damage to the bladder(Y/N) & $13 / 116(11.2 \%)$ \\
Damage to the Ureter & 0 \\
Fever & $9 / 116(7.8 \%)$ \\
\hline
\end{tabular}

* Myomectomy, tumor of ovary, or tubal ligation. 


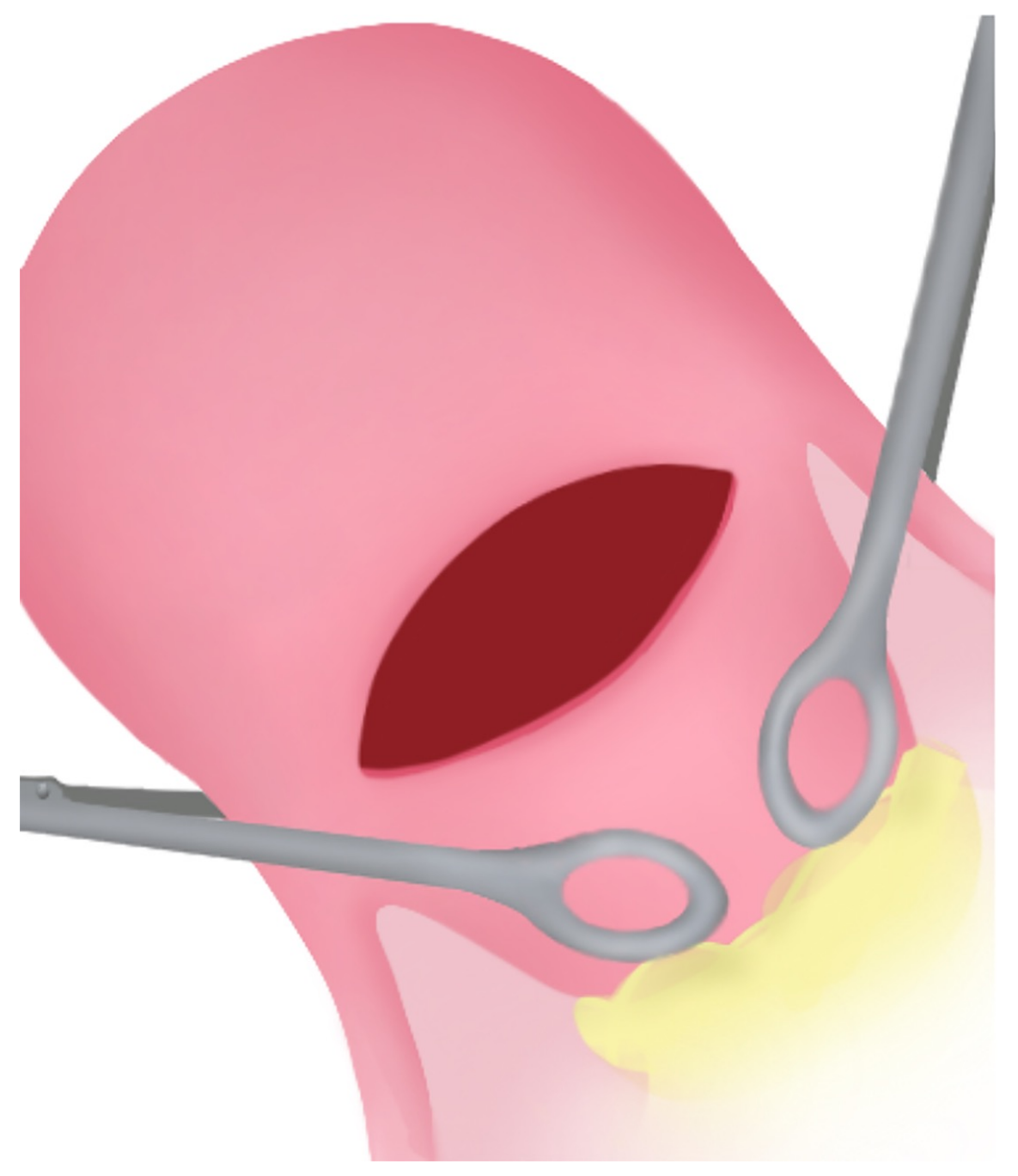




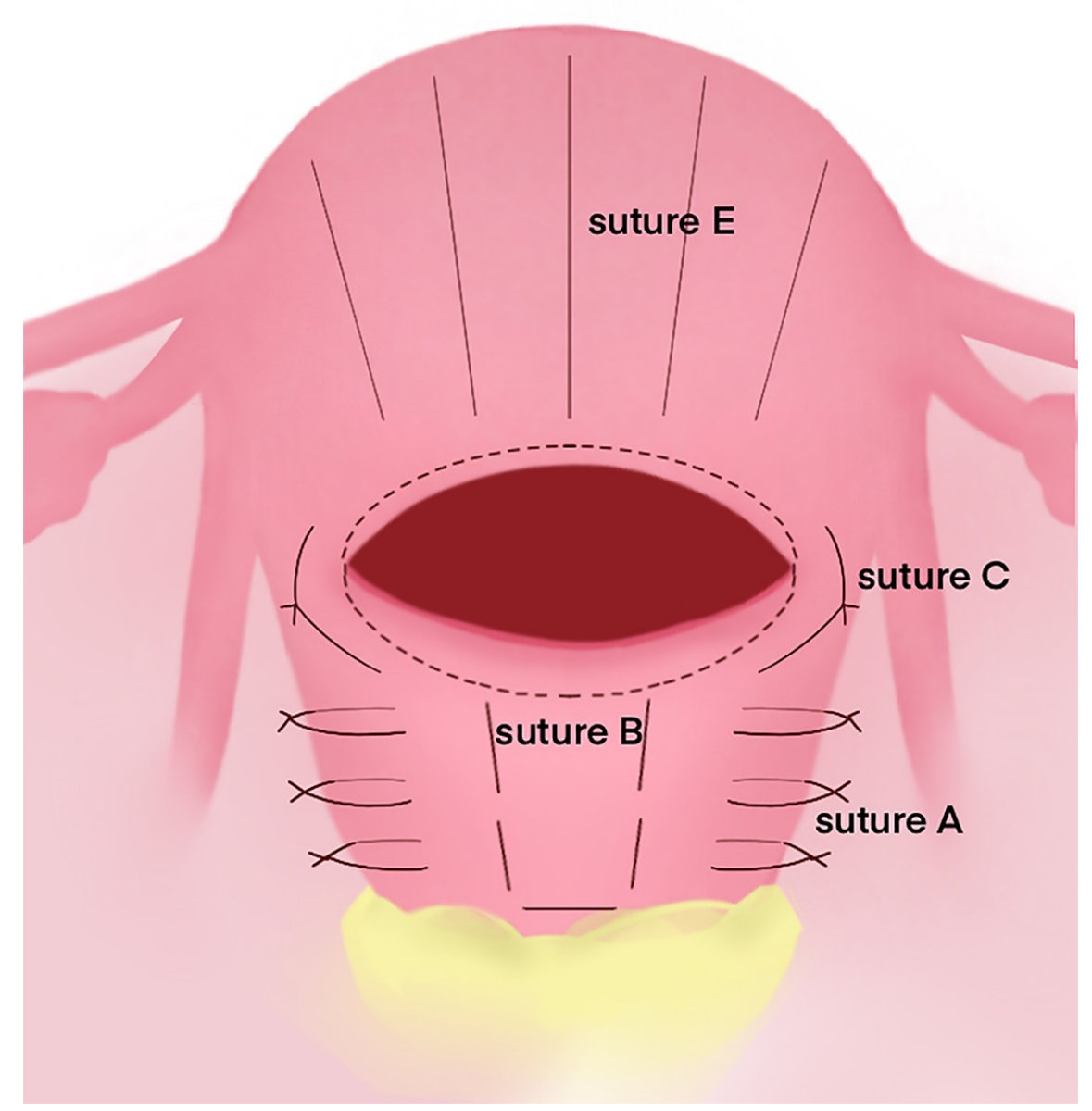




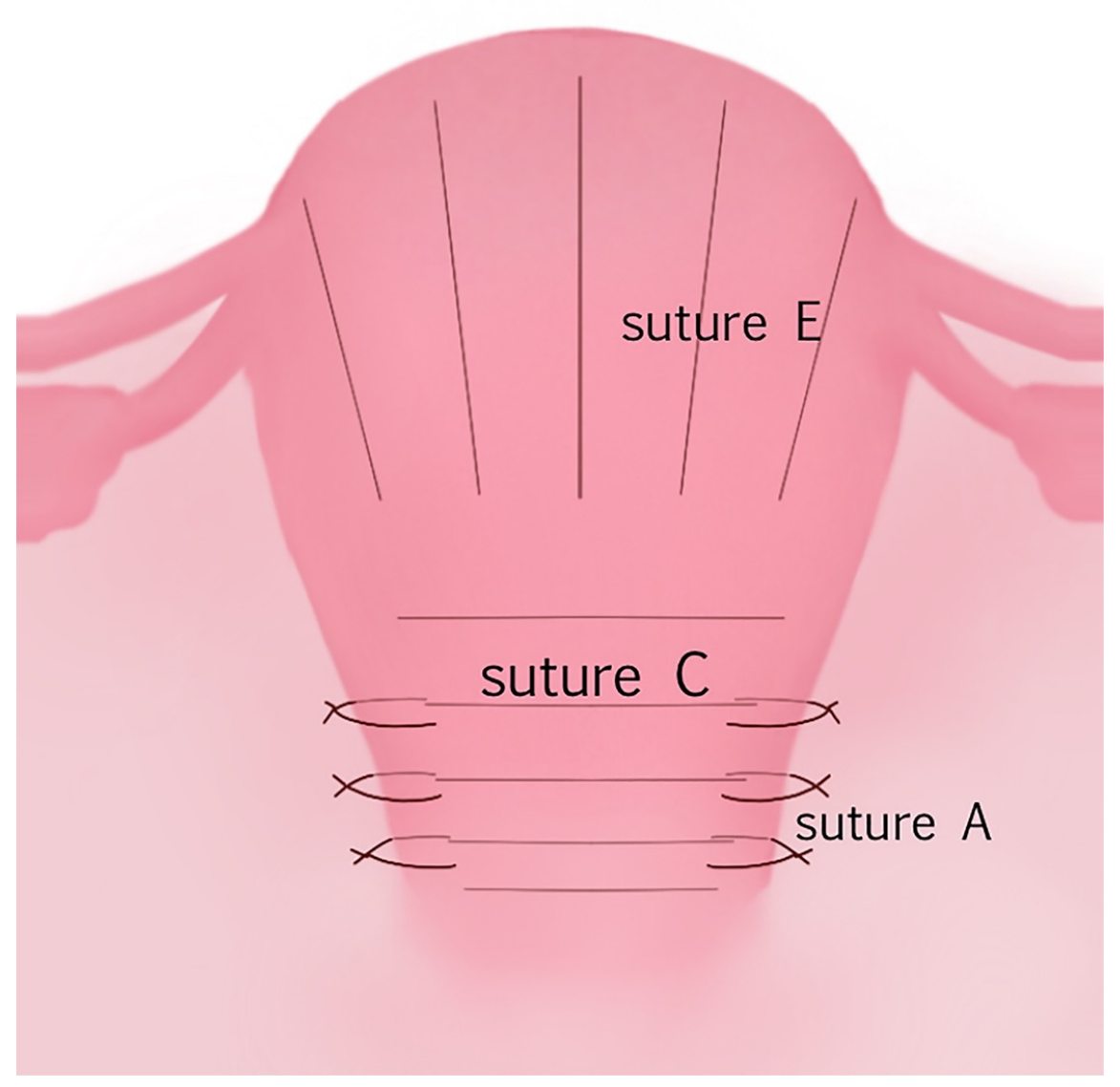

